\title{
Maximal Area Sets and Harmony
}

\author{
David Rappaport ${ }^{1}$ \\ School of Computing, Queen's University \\ Kingston, ON CANADA
}

\begin{abstract}
A musical scale can be viewed as a subset of notes or pitches taken from a chromatic universe. For the purposes of this paper we consider a chromatic universe of twelve equally spaced pitches. Given integers $(N, K)$ with $N>K$ we use particular integer partitions of $N$ into $K$ parts to construct distinguished sets, or scales. We show that a natural geometric realization of these sets have maximal area, so we call them maximal area sets. We then discuss properties of maximal area sets for the integer pairs $(12,5)(12,6)(12,7)$ and $(12,8)$ with the obvious relevance to scales in our normal chromatic collection of 12 pitches. Complementary maximal area sets are those sets where the chosen $K$ notes realize maximal area, and the complementary $N-K$ notes also realize maximal area. The complementary maximal area sets closely match a significant collection of scales identified in a book on jazz theory by Mark Levine [9].
\end{abstract}

Key words. Geometry, Harmony, Scale, Integer partition, Necklace.

\section{Introduction}

Geometry and music are intertwined in many different ways. Music notation uses shape and space to convey pitch and time information. Guitar players visualize harmonic structures such as scales, arpeggios and chords, as geometric shapes on the fret-board. The origins of our musical system of seven-note scales chosen from a collection of 12 pitches may be described in terms vibrating strings of various lengths. Recently, in a paper by Dimitri Tymoczko [17], geometry is used to reason about voice leading techniques. Combinatorics is another branch of mathematics that is used in music analysis. Inevitably combinatorial insight is supported by a picture, that is, a geometric representation.

Consider a circle with twelve equidistant points spread out on its boundary. The twelve points represent the 12 equally spaced pitches that represent the chromatic universe using an equal tempered tuning. From these 12 points we choose a subset of at least five points, because musically a subset of five or more pitches are called scales. Some of these subsets, or scales, are at the centre of western harmony.

In the examples shown in Figure 1, a subset of points are connected in sequence to construct a convex polygon. We consider distinct polygons up to rotation. This corresponds to the notion that different modes from the same scale are not different scales.

Since there are twelve equally spaced markings on the circle it makes sense to call these diagrams clock diagrams. Representing the notes of a scale by a polygon appears 


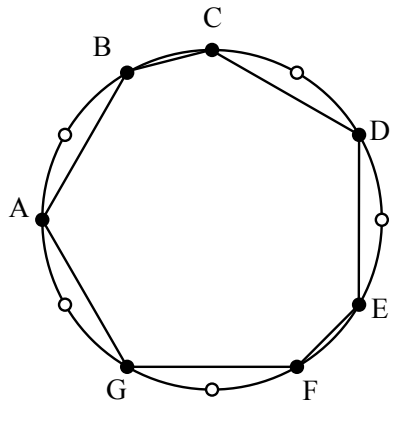

a

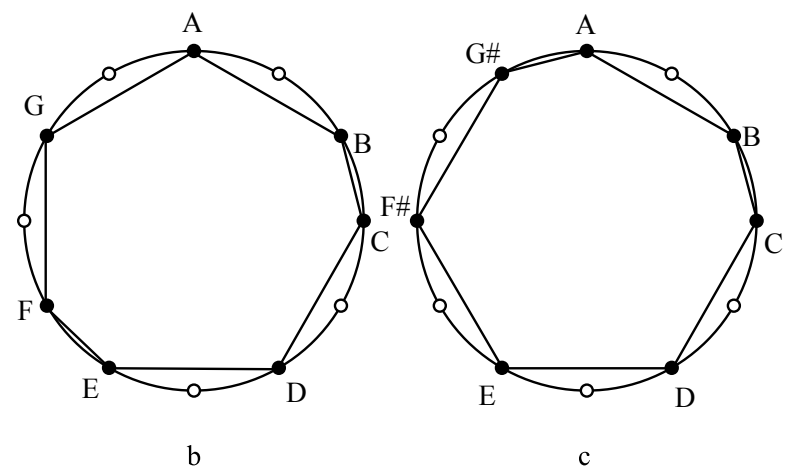

Fig. 1. The subsets in a) and b) represent two modes of the diatonic scale, Ionian and Aeolian, also known as the major and natural minor scales. For our purposes these two scales are considered to be equivalent. The diagram of part c) represents the ascending melodic minor scale and is distinct from a) and b).

in a paper published in 1937 by E. Krenek [8], so sometimes these diagrams are called Krenek diagrams as in the paper by McCartin [10]. However, in an account by Nolan [11], Heinrich Vincent used this very same representation in his paper published in 1862 [18]. The use of clock diagrams is ubiquitous in mathematical music theory. When looking at the notes of the usual diatonic scale, observe that they are spread out evenly amongst the twelve chromatic pitches. The distance between two notes can be measured as the number of scale notes between them, or the total number of notes, scalar or not, between them. In this way we can distinguish between the scalar distance from the chromatic distance of a pair of notes. Clough and Douthett [1] define a set to be maximally even if the chromatic distance between two pairs of notes of the same scalar distance differs by at most one. The maximally even sets (ME sets) are unique (up to rotation) as proved in [1] and also in [4]. The ME sets include some of the most widely used scales in Western music, that is, the diatonic scale, the common anhemitonic pentatonic scale, the six note whole-note scale, and the eight note diminished scale.

When ME sets are represented by a clock diagram, then those points are subsets which uniquely maximize the sum of inter-point distances [2-4]. A similar continuous case of this phenomenon is described by Fejes Tóth [14]. In that paper it is shown that a finite set of $N$ points that maximize the sum of inter-point distances are located on the vertices of a regular convex $N$-gon. That is, the points are spread out as evenly as possible on the circumference of a circle.

In his book on harmony for the improvising jazz musician Levine [9] describes four fundamental scales that are useful for jazz improvisation. These four scales are the sevennote major scale, the seven-note melodic minor scale, the six-note symmetric whole-tone scale and the eight-note diminished scale. In jazz terminology the term "melodic minor" almost always denotes the ascending melodic minor scale, and we follow this convention.

Three of these four scales are maximally even, the exception being the melodic minor scale which is not. Thus given pairs $(12,8)(12,6)$ and $(12,7)$ we may ask whether there is a mathematical characterization that exactly describes Levine's four fundamental scales. In this note we arrive at such a characterization called complementary maximal area sets.

The paper is organized as follows. In the next section we provide a mathematical discussion on a class of subsets of $K$ elements chosen from $N$. This characterization is 

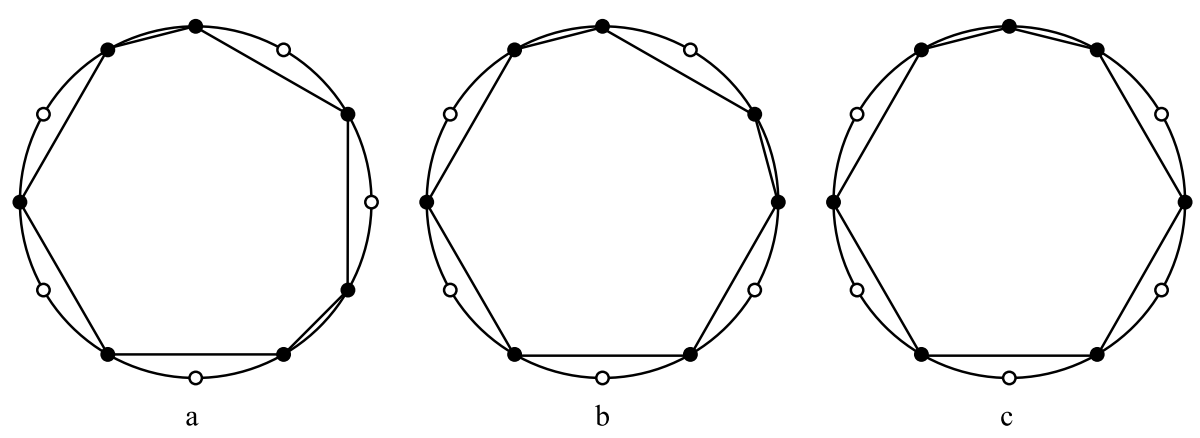

Fig. 2. Clock diagrams of the three MA scales. The interval structure of these scales are (a) the diatonic scale, (b) the ascending melodic minor and (c) the Neapolitan major scale.

both combinatorial and geometric. We begin by describing the so called maximal area sets, and prove some mathematical properties of these sets. The maximal area sets are interesting in their own right, but do not quite satisfy the goals mentioned above, as this characterization includes subsets of $(12,8)$ and $(12,7)$ that are not from the four fundamental scales. In section 3 we then define and analyze complementary maximal area sets and show that this characterization satisfies our requirements. The paper concludes with some closing remarks in section 4.

\section{Maximal Area Sets}

A common misconception is that the prefix $d i$ in diatonic refers to the number two, signifying the characteristic that there are two step sizes in the usual diatonic set. However, the truth is the prefix dia refers to from the tonic [12]. Nevertheless, this definition is the ideal spring board from which we can launch an exploration of scales that satisfy this property, that is collections of subsets of 7 pitches from 12, so that the spaces between consecutive pitches are either whole tones or semitones. This results in three distinct scales. Using clock diagrams we show these three distinct scales in Figure 2. In (a) we recognize the standard diatonic scale, (b) represents the melodic minor and in (c) we have the symmetric whole-note scale plus a note, also called the Neapolitan major scale.

It is not hard to verify that the polygons representing the scales have equal area, and this area is maximized for any choice of seven points from twelve. Thus we will refer to these scales as maximal area scales, or more generically maximal area subsets which we abbreviate as MA sets.

We generalize this notion for any subset of $K$ pitches chosen from a chromatic universe of $N$ pitches. It will be most convenient to define our subsets in terms of integer partitions.

An integer partition of a natural number $N$ is a way of writing $N$ as an unordered sum of natural numbers. In [7] Keith points out the connection between integer partitions and musical scales.

Definition: A set of $K$ pitches taken from a chromatic universe of $N$ pitches numbered $1 \ldots N$, is an $M A$ set if it satisfies the following properties.

- There is an integer partition of $N$ using exactly $K$ positive integer summands, that is, $N=a_{1}+a_{2}+\cdots+a_{K}$.

- The summands differ by at most one, that is, $\left|a_{i}-a_{j}\right| \leq 1$, for all $i, j$.

The following proposition provides a mathematical foundation for constructing and analyzing MA sets. 
Proposition 1. Given integers $N, K$ with $K<N$, there exist unique integers $u$ and $m$ such that $N=m u+(K-m)(u+1)$.

Observe that for $N, K, U, m$ as defined above we have the integer partition $N=a_{1}+$ $a_{2}+\cdots+a_{K}$ with $a_{i}=u$, for $i=1 \ldots m$ and $a_{i}=u+1$, for $i=m+1 \ldots K$. Here we have the understanding that $i=m+1 \ldots K$ is the empty set in the event that $m=K$, that is $K$ divides $N$.

Proof: Let

$$
u=\left\lfloor\frac{N}{K}\right\rfloor \text { and } v=\left\lceil\frac{N}{K}\right\rceil .
$$

Note that if $K$ divides $N$ then $v=u$, otherwise $v=u+1$.

For the case $v=u$ we have $N=K u$. Considering the case where $v=u+1$ we have the equality $(N-K u) v+(K v-N) u=N(v-u)=N$. Thus $m=K v-N=K(u+1)-N$. Since $u$ determines $m$ it suffices to show that $u$ is the unique value satisfying the required conditions. When $K$ divides $N$ uniqueness follows from the division algorithm [6]. When $K$ does not divide $N$ we enumerate the cases of using a number larger or smaller than $u$. Thus let $w$ be an integer and $w>\left\lfloor\frac{N}{K}\right\rfloor$. However, this implies that $K w>N$ so $w$ cannot be greater than $u$. A similar symmetric argument can be used to show that $w<\left\lfloor\frac{N}{K}\right\rfloor$ leads to a contradiction.

Thus we have shown that $u$ is unique, completing our proof.

Recall that in ME sets as defined by Clough and Douthett $[2,3]$ the chromatic distance between two pairs of notes of the same scalar distance differs by at most one. This immediately leads to the following proposition.

Proposition 2. If a set is an ME set then it must also be an MA set.

As was shown by the example illustrated in Figure 2 that although for any $N, K$ there are unique values of $u, m$, one can possibly obtain more than one scale with step sizes $u$ and $u+1$ by reordering the positions of the $u$ steps with respect to the $u+1$ steps. Given $(N, K, u, m)$ we can enumerate the distinct number of scales (up to rotation) that are MA scales. This value depends only on $K$ and $m$, and is the number of distinct $K$-ary necklaces that are obtained using two types of beads, $m$ white and $K-m$ black, where a $K$-ary necklace is defined as an equivalence class of $K$-ary strings under rotation, see [13]. Distinct necklaces can be enumerated in $\mathrm{O}(K)$ time per necklace using an algorithm due to Sawada and Ruskey [13].

We now turn to the question of the area of the representative polygons. Referring to Figure 3 it is clear that the area of the septagon is obtained by summing triangle areas.

Assuming that the septagon representing these scales is circumscribed by a unit radius circle, a formula giving the polygon area is: $\sin (\pi / 6)+5 / 2 \sin (\pi / 3)$.

In general given $(N, K, u, m)$ the area of the representative polygons can be found by summing the area of the triangles that partition the polygon. For our purposes it is most convenient to partition the polygon into triangles that share a common vertex at the centre of the circumscribing circle and whose sides are radii. From now on we refer to this particular partition as the triangle partition of the polygon. The sum of the areas of the triangles in any polygon representation of $(N, K, u, m)$ is given by the formula:

$$
\frac{m}{2} \sin \left(\frac{2 \pi u}{N}\right)+\frac{K-m}{2} \sin \left(\frac{2 \pi(u+1)}{N}\right) .
$$




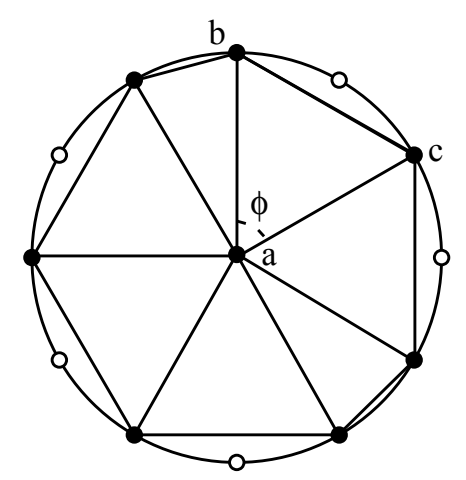

Fig. 3. One can obtain the area of the septagon by summing the areas of triangles in the traingle partition as suggested above. The area of the triangle $a, b, c$ is given by $\sin (\phi) / 2$. The perimeter of the inscribed polygon is also a function of the centre angles. For example the length of the polygon edge $b c$ is $\sin (\phi / 2)$

Note that the area is a function that only relies on the values of the angles of the triangles found at the centre of the circle. We refer to these angles as centre angles.

We claim that all of these septagons are area maximizing septagons. This is easy enough to verify for this example. We prove the result for the general case in the next lemma. Furthermore, we show that these polygons also maximize perimeter. The fact that perimeter is also maximized is realized when we see that the perimeter is also a function that relies only on the centre angles. The formula for the perimeter of polygon representations of $(N, K, u, m)$ is given by the formula:

$$
2 m \sin \left(\frac{u \pi}{N}\right)+2(K-m) \sin \left(\frac{(u+1) \pi}{N}\right) .
$$

Lemma 1. Given $(N, K, u, m)$ the polygon representations of these $M A$ sets have maximum area and maximum perimeter.

Proof: Consider a $k$-gon $X$ that is not a representation of an MA set. Thus there are two triangles in the triangle partition of $X$ that have centre angles $\alpha_{1}$ and $\alpha_{2}$ and the difference $\alpha_{1}-\alpha_{2} \geq 2 / n$. Let us make the mild assumption that $\alpha_{1}<\alpha_{2}<\pi$. Letting $\alpha_{1}+\alpha_{2}=A$, we have

$$
A / 2 \geq \alpha_{1}+2 \pi / n .
$$

Since the ordering of the triangles has no effect on the area or perimeter of the polygon we can arrange it so that these two triangles are adjacent. We can write the sum of the area of these two triangles as $1 / 2\left(\sin \left(A-\alpha_{1}\right)+\sin \left(\alpha_{1}\right)\right)$. If we take the first derivative of the area with respect to $\alpha_{1}$, that is, $1 / 2\left(\cos \left(\alpha_{1}\right)-\cos \left(A-\alpha_{1}\right)\right)$, and set it to zero we see that a value of $\alpha_{1}=A / 2$ is maximizing. Also the first derivative is positive for all values of $0 \leq \alpha_{1} \leq A / 2$.

Let $\alpha_{1}^{*}=\alpha_{1}+2 \pi / n$ and $\alpha_{2}^{*}=\alpha_{2}^{*}-2 \pi / n$. By Equation 1 we see that $\alpha_{1}^{*} \leq A / 2 \leq \alpha_{2}^{*}$. Thus the new area sum is greater and $X$ cannot have maximal area.

For perimeter we use a similar argument. The sum of the polygon edges are given by the equation $2\left(\sin \left(\left(A-\alpha_{1}\right) / 2\right)+\sin \left(\alpha_{1} / 2\right)\right)$, and its first derivative is $\cos \left(\alpha_{1} / 2\right)-\cos ((A-$ $\left.\alpha_{1}\right) / 2$ ). We see again that the sum is maximized when $\alpha_{1}=A / 2$, and this derivative is positive for $0 \geq \alpha_{1} \geq A / 2$. Again we let $\alpha_{1}^{*}=\alpha_{1}+2 \pi / n$ and $\alpha_{2}^{*}=\alpha_{2}^{*}-2 \pi / n$. By Equation 1 we see that $\alpha_{1}^{*} \leq A / 2 \leq \alpha_{2}^{*}$, and $X$ cannot have maximal perimeter. 

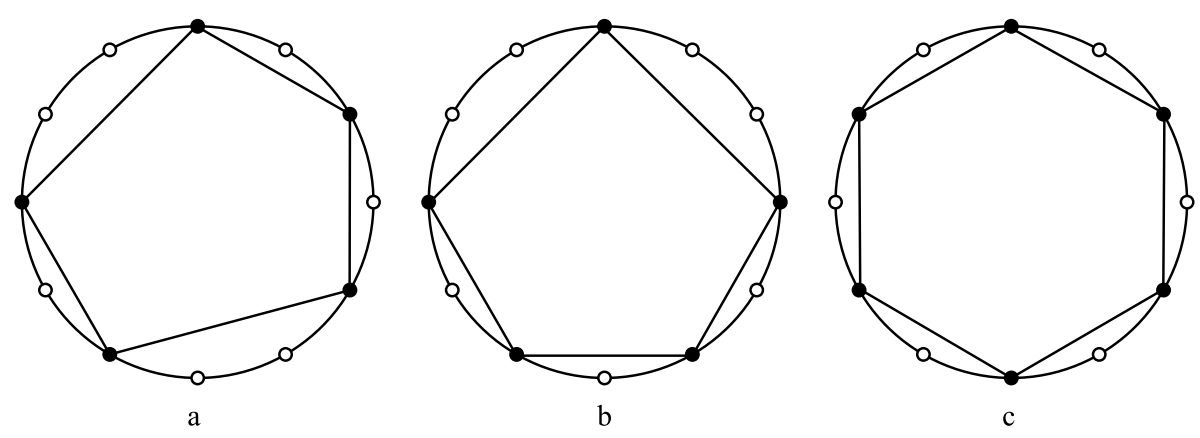

Fig. 4. The five and six-note maximal area sets.

\section{Complementary Maximal Area Sets.}

Four scales that are distinguished by Levine [9, Chapter 3] in the "Chord/Scale" chapter in his book on jazz harmony are the symmetric whole-tone scale, the major scale, the melodic minor scale, and the diminished scale. We define a class of scales, the complementary maximal area (CMA) scales, so that for the integer pairs $(12,6),(12,7)$, and $(12,8)$ correspond identically to the four scales distinguished by Levine.

Definition: A set of $K$ pitches taken from a chromatic universe of $N$ pitches numbered $1 \ldots N$, is a $C M A$ set if it satisfies the following properties.

- The set is an MA set.

- The $N-K$ notes that are in the complementary set are also an MA set.

We showed that ME sets are MA sets. ME sets are also CMA sets because the complement of an ME set is also an ME [1] set. Thus the CMA scales are a (strictly) larger family than the ME scales.

There is a single $(12,6)$ MA set, the symmetric whole-tone scale, shown in Figure 4 c). Clearly this scale is its own complement and thus is a CMA set . Of the three $(12,7)$ MA sets two have complements that are MA sets. These $(12,5)$ CMA scales are shown in Figure 4 .

There are ten $(12,8)$ MA sets, as described in [7, p. 31], we reproduce them in figure Figure 5. There is only one of these MA sets whose complementary set is also an MA set. In Figure 6 we show this set and its four note complement.

Thus we have been able to capture a mathematical property that characterizes Levine's four fundamental scales.

\section{Discussion}

We have shown that a particular integer partition of $N$ into $K$ parts leads to maximal area polygons when these sets are represented with a clock diagram. These so called maximal area sets are computationally easy to find. However, a classification that seems more interesting uses the complementary maximal area sets. We have demonstrated that the complementary maximal area sets for $(12,6)(12,7)$ and $(12,8)$ contain the four fundamental scales as defined by Levine in his book on jazz improvisation. These fundamental four scales by no means exhaust the large number of scales that are regularly used by jazz musicians.

In a separate chapter Levine discusses pentatonic scales and their role in jazz improvisation. By far the most important of the five-note scales is the common anhemitonic 

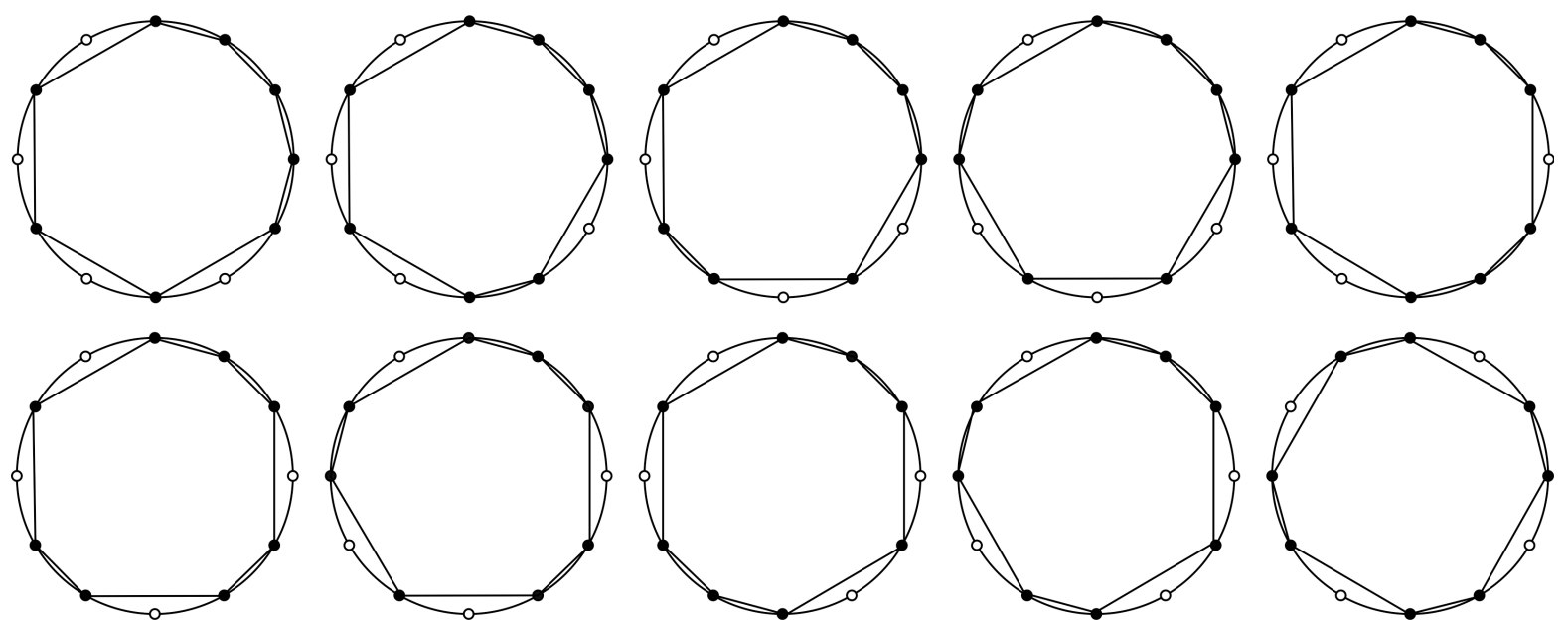

Fig. 5. The 10 eight-note maximal area sets.
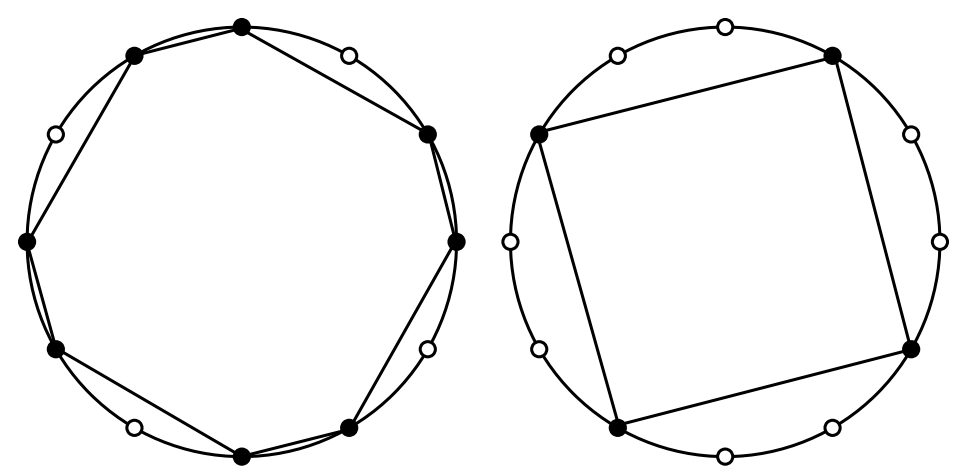

Fig. 6. The single complementary maximal area eight-note scale (the diminished scale) with its complement (a diminished seventh chord).

pentatonic scale, which we already know to be a CMA scale. There is one more collection of five notes that make a CMA set and they are shown in Figure $4 \mathrm{~b}$ ). This scale can be called a dominant pentatonic scale, as it contains a dominant chord, however, this scale seems to be obscure, and is not mentioned at all in Levine's book.

If we consider a rhythmic analog of the clock diagrams, that is, selected points represent onsets of beats, then the five element CMA sets shown in Figure 4 represents the Flamenco hand clapping pattern used in the Solea, Buleria, and Guajira, see [5].

Acknowledgements. I am pleased to acknowledge the papers of Godfried Toussaint, [15, 16] as my first encounter with the use of clock diagrams to represent musical pitch and/or musical rhythm. I would also like to acknowledge two anonymous referees who provided useful suggestions that I think has improved the readability of this paper.

\section{References}

1. J. Clough and J. Douthett: Maximally even sets. Journal of Music Theory, 35, (1991) 93-173.

2. J. Clough and G. Myerson: Musical scales and the generalized circle of fifths. American Mathematical Monthly 93:9 (1985) 695-701. 
3. J. Clough and G. Myerson: Variety and multiplicity in diatonic systems. Journal of Music Theory 29 (1985) 249-270.

4. Erik D. Demaine, Francisco Gomez-Martin, Henk Meijer, David Rappaport, Perouz Taslakian, Godfried T. Toussaint, Terry Winograd, and David R. Wood: The distance geometry of music. submitted to Computational Geometry: Theory and Applications, (2006).

5. Miguel Díaz-Bañez, Giovanna Farigu, Francisco Gómez, David Rappaport, and Godfried T. Toussaint: El compás flamenco: a phylogenetic analysis. Proceedings of BRIDGES: Mathematical Connections in Art, Music and Science, Winfield, Kansas (2004) 61-70.

6. Ralph Grimaldi: Discrete and Combinatorial Mathematics: An Applied Introduction. Addison Wesley (1998).

7. Michael Keith: From Polychords to Polya: Adventures in Musical Combinatorics. Vinculum Press, Princeton (1991).

8. E. Krenek: Über Neue Musik. chapterMusik und mathematik. Verlag der Ringbuchhandlung, Vienna (1937) 71-89.

9. Mark Levine: The Jazz Theory Book. Sher Music Co. (1995).

10. Brian J. McCartin: Prelude to musical geometry. The College Mathematics Journal, 29:5 (1998) 354-370.

11. Catherine Nolan: Combinatorial space in nineteenth- and early twentieth-century music. Music Theory Spectrum, 25:2 (2003) 205-241.

12. D. Randel (editor): The Harvard Dictionary of Music. Harvard University Press (1986).

13. J. Sawada and F. Ruskey: An efficient algorithm for generating necklaces with fixed density SIAM Journal on Computing 29:2 (1999) 671-684.

14. L. Fejes Tóth: On the sum of distances determined by a pointset. Acta. Math. Acad. Sci. Hungar. 7:3 (1956) 97-101.

15. Godfried T. Toussaint: A mathematical analysis of African, Brazilian and Cuban clave rhythms. Proceedings of BRIDGES: Mathematical Connections in Art, Music and Science, Towson, Maryland (2002) 157-168.

16. Godfried T. Toussaint: Classification and phylogenetic analysis of African ternary rhythm timelines. Proceedings of BRIDGES: Mathematical Connections in Art, Music and Science, Granada, Spain (2003) 25-36.

17. Dmitri Tymoczko: The Geometry of Musical Chords. Science 313 (2006) 72-74.

18. Heinrich Vincent: Die Einheit in der Tonwelt. Verlag von Heinrich Matthes, Leipzig (1862).

Received:

Final version received: 